\title{
Asteroseismology in Open Clusters
}

\author{
S. Frandsen and M. Viskum \\ Institute of Physics and Astronomy, Aarhus University, Denmark \\ M.M. Hernandéz and J.A. Belmonte \\ Instituto de Astrofisica de Canarias, Tenerife, Spain
}

\section{A stellar evolution test}

Within two groups or networks we are trying to build the observational fundamentals for better tests of stellar evolution. The first group is STACC (Small Telescope Array with CCD Cameras) and the second the EU HCM program ANTENA (involving institutes in Spain, France and Denmark).

The first information needed is photometric data. Colour-Luminosity diagrams and time series photometry must be obtained. The time series require extensive campaigns in order to give the high quality data needed for asteroseismology. Ideally one should have time series of several quantities (see Balona \& Stobie 1979, Mantegazza et al. 1994).

The second type of observations is of spectroscopic nature. It is needed to ascertain membership of the stars in a given cluster and to check binarity. Also rotational velocities must be observed to help the attempt to identify the modes in $\delta$ Scuti stars. Finally some information about metallicity and peculiarity is required.

With a complete set of data for a cluster, many normal degrees of freedom disappear and a very stringent test of several aspects of stellar models becomes feasible.

In addition some specific questions about $\delta$ Scuti stars can be addressed like the identification of modes, the excitation and the period and amplitude changes reported (Breger 1990).

\section{A definition of a good open cluster}

\section{Distance}

- $d \geq 1 \mathrm{Kpc}$ : The angular size of the cluster on the sky must fit into the field of view of the telescope/CCD system typically available.

- $d \leq 2 \mathrm{Kpc}$ : The precision of the time series studies needed prevents small telescopes from being used for distant clusters. Crowding becomes worse and influences the measurements even if large telescopes are used. Spectroscopic information becomes very expensive in terms of telescope allocation: Long exposures on large telescopes. 
- $8.5<\log t<9.0$ Younger clusters only have main sequence $\delta$ Scuti stars with very low amplitudes and therefore hard to locate and hard to obtain good precision for. Older clusters simply have turnoff below the instability region. The best clusters have a population of giants, which is useful, when the age and metallicity of the cluster have to be determined.

Metal abundance

- $0.3 \geq[\mathrm{Fe} / \mathrm{H}] \geq-0.3$ Although it probably does not remove any cluster from the list of targets a nearly solar abundance is preferable. But, open clusters normally satisfy this demand without problem.

\subsection{Open clusters under study}

Table 1 presents a range of clusters with measurements of very different extent from just a few frames up to thousands in the case of NGC 6134. The lack of variables in some of the clusters does not necessarily reflect a true deficit of variables. It is as much a function of the amount of data available for the clusters.

Table 1. Open clusters with CCD measurements.

\begin{tabular}{lrrrc} 
Cluster & Decl. & Dist.(pc) & $\log ($ Age $)$ & Comments $^{a}$ \\
\hline NGC 2099 & $+32^{\circ}$ & 1350 & 8.48 & \\
NGC 2194 & $+13^{\circ}$ & 2700 & 8.57 & \\
NGC 2251 & $+8^{\circ}$ & 1550 & 8.48 & \\
NGC 2355 & $+13^{\circ}$ & 1600 & 8.99 & \\
NGC 2660 & $-47^{\circ}$ & 2680 & 9.22 & $4 \delta^{\prime}$ 's, 1 EB \\
NGC 6134 & $-49^{\circ}$ & 900 & 8.90 & $7 \delta^{\prime}$ 's 1 BS \\
NGC 7062 & $+46^{\circ}$ & 1700 & 8.69 & $3-5$ 's \\
NGC 7209 & $+46^{\circ}$ & 900 & 8.50 & \\
NGC 7226 & $+55^{\circ}$ & 2100 & 8.34 & $2 \delta$ 's, 1 EB \\
NGC 7245 & $+54^{\circ}$ & 1850 & 8.19 & $2 \delta$ 's, 1 EB, 1 W UMA \\
NGC 7654 & $+61^{\circ}$ & 1600 & 8.23 & $1 \delta, 1$ EB, 1? \\
\hline
\end{tabular}

${ }^{a}$ The explanation of signatures is EB: Eclipsing Binary, BS: Blue Straggler, $\delta$ : $\delta$ Scuti star, W UMA: Contact binary

In order to succeed in the search the procedures described by Kjeldsen \& Frandsen (1992), Gilliland \& Brown (1994) and Frandsen et al. (1995) should be followed in detail.

\section{References}

Balona, L. A., \& Stobie, R. S. 1979, MNRAS, 189, 649

Breger, M. 1990, in "Confrontation between stellar pulsation and evolution", (eds.) C. Cacciari \& G. Clementini, ASP Conf. Ser. 11, 263

Gilliland, R. L., \& Brown, T.M. 1994, ARA\&A, 32, 37

Frandsen, S., Jones, A., Kjeldsen, H., et al. 1995, A\&A, in press

Kjeldsen, H., \& Frandsen, S. 1992, PASP, 104, 413

Mantegazza, L., Poretti, E., \& Bossi, M. 1994, A\&A, 287, 95 